\title{
Factores determinantes de la rentabilidad de las granjas de vacuno de leche en Castilla y León
}

\author{
María José Fernández de la Cal11, ${ }^{1,}$ Ángel Ruiz Mantecón² y Alfonso Moral ${ }^{3}$ \\ 1 Universidad de Valladolid, Programa de doctorado, Av. Valle de Esgueva, 6, 47011 Valladolid \\ 2 Instituto de Ganadería de Montaña, CSIC-Universidad de León, Finca Marzanas, 24346 Grulleros, \\ León, España \\ 3 Universidad de Valladolid, Facultad de Ciencias Sociales, Jurídicas y de la Comunicación, Plaza Univer- \\ sidad No 1, 47005 Segovia, España
}

\section{Resumen}

Este trabajo pretende analizar los factores determinantes de la rentabilidad de las explotaciones de vacuno lechero en la región de Castilla y León. La rentabilidad se ha medido a través de dos indicadores: el beneficio por vaca y la producción umbral. Se ha utilizado la información correspondiente a un grupo de granjas de vacuno de leche, en las que se tomaron datos durante los años 2016, 2017 y 2018. De cada una de estas explotaciones se obtiene información correspondiente a censos de ganado, estructura de inmovilizado material, ingresos, costes y otras variables que han permitido evaluar su situación económico-financiera y construir indicadores que determinan sus resultados económicos.

Los datos han sido analizados mediante una estimación mínimo cuadrática de dos especificaciones econométricas multivariantes, una para cada uno de los indicadores de rentabilidad analizados. Los resultados ponen de manifiesto la importancia que tienen algunos componentes del coste y de los ingresos para explicar la rentabilidad de las granjas. También se observan efectos relevantes del tamaño de la explotación, de los controles anuales y de las variables que indican la localización territorial de la explotación.

Palabras clave: Vacuno lechero, rentabilidad, beneficio, producción umbral, Castilla y León.

\section{Determining factors of the profitability of dairy farms in Castilla y León}

\section{Abstract}

This work pursues a twofold objective, on the one hand a database of dairy farms is elaborated and, on the other hand, the factors determining their profitability are analysed through two indicators: the benefit per cow and the threshold production. For the first objective we took a representative sample of 30 dairy farms located in the autonomous community of Castilla y Leon during the years 2016, 2017 and 2018. From each of these farms we obtained data regarding livestock censuses, structure of tangible assets, income, costs and other variables that allow us to assess their economic-financial situation and to build the profitability indicators.

To achieve the second objective, an ordinary least squares estimation over two multivariate econometric specifications was made, one for each indicator. In this way it is possible to obtain the ceteris paribus

\footnotetext{
* Autor para correspondencia: mariajose.fernandez.cal@alumnos.uva.es
}

Cita del artículo: Fernández de la Cal MJ, Mantecón AR, Moral A (2021). Factores determinantes de la rentabilidad de las granjas de vacuno de leche en Castilla y León. ITEA-Información Técnica Económica Agraria 117(4): 360-374. https://doi.org/10.12706/itea.2020.040 
effect of each explanatory variable included in the regression. Results show the importance of some cost and income components to explain firms' profitability. There are also relevant effects of the farm size, annual controls or provincial dummy variables indicating the place where the firm is located.

Keywords: Milk beef farm, economic profitability, multivariate analysis, threshold production, Castilla y León.

\section{Introducción}

La producción de leche de vaca puede ser considerada como una actividad estratégica por su importancia dentro del PIB del sector primario y su relevancia a la hora de fijar de población en zonas rurales. De acuerdo con los datos correspondientes a la renta agraria nacional del año 2018 que proporciona el INE (INE, 2018), la producción de leche suponía casi el $6 \%$ de toda la producción agraria y superaba el $16 \%$ de la producción final ganadera de España. Esos porcentajes se situaban en torno al $11 \%$ y $18 \%$ en el caso de Castilla y León para el año 2017 (último disponible). Más allá de su importancia relativa, se trata de un sector sometido a crisis periódicas debido a la volatilidad de los precios que las industrias pagan por la leche y de las materias primas usadas para la alimentación del ganado (RENGRATI, 2018). Esta situación de riesgo para las inversiones sirve como primera justificación para la realización de este tipo de estudios.

A nivel internacional, el sector del vacuno de leche en España ocupaba la séptima posición en producción de leche dentro de la UE en 2018 con más de 7 millones de toneladas de producción industrial de todo tipo de leche. $Y$ esto en un marco de crecimiento europeo en la producción láctea que ha sido sostenido durante los años a los que pertenece este análisis y que en 2018 superaba los 155 millones de toneladas (MAPA, 2020). Por su parte, Castilla y León era la segunda productora de leche de España durante ese año, con una cuota del $13 \%$ y más de 900 millones de litros, solo superada por Galicia. De acuerdo con el in- forme de coyuntura del sector vacuno de leche de abril de 2019, las explotaciones de ganado vacuno lechero en Castilla y León en los últimos años se caracterizan por un aumento de tamaño y de la producción individual de las vacas (MAPA, 2019). Esta evolución, y las importantes inversiones que acarrea, colocan a los análisis de rentabilidad como un elemento esencial para garantizar la sostenibilidad de las explotaciones.

La información utilizada procede de una base de datos de granjas a las que se ha realizado un seguimiento durante los años 2016, 2017 y 2018. En total se utiliza información de entre 26 y 28 explotaciones (dependiendo del año) localizadas en Castilla y León. Esta es otra de las aportaciones de este trabajo, por ser la primera vez que se construye una muestra de estas características para esta comunidad.

Con estos antecedentes, el trabajo plantea un análisis multivariante que trata de identificar los factores determinantes de la rentabilidad de las granjas analizadas. Este tipo de análisis ha adquirido importancia en la literatura sobre el tema desde diferentes puntos de vista. Por un lado, existen informes muy completos realizados por el ministerio sobre resultados técnicos y económicos de granjas (ECREA, 2016 y 2017). Desde una perspectiva más académica Pérez-Cabal y Alenda (2003), Acero et al. (2004) y Álvarez et al. (2016) realizan estudios en esta línea para el ganado vacuno y Milán et al. (2014) para el caso del ovino. Finalmente, este tipo de análisis también ha tenido trascendencia a nivel internacional como lo muestran los trabajos de Nehring et al. (2016) con granjas americanas o europeas y Sinisalo (2015) en Finlandia. 
En este trabajo se analiza la magnitud y significatividad del efecto que la composición de los costes corrientes, el origen de los ingresos, el grado de mecanización de la empresa, la importancia de las subvenciones o el tamaño de la explotación tienen sobre sus resultados económicos. También se tiene en cuenta el posible efecto de la localización territorial de la explotación o la dimensión temporal de la información. El estudio se realiza para dos posibles proxis de la rentabilidad de la granja, el beneficio por vaca y el umbral de producción que es necesario obtener por vaca para cubrir los costes corrientes. Estos dos indicadores son comunes en los análisis realizados por la Red Nacional de Granjas Típicas (RENGRATI) y se calculan por vaca para permitir la comparación entre explotaciones. En esta misma línea, los informes ECREA también utilizan euros por vaca como referencia a la hora de presentar algunos de sus indicadores.

\section{Material y métodos}

Desde un punto de vista meramente geográfico Castilla y León como área de estudio tiene un promedio de Altitud de $830 \mathrm{~m}$ sobre el nivel del mar (500 m a $2.500 \mathrm{~m} \mathrm{s.} \mathrm{n.} \mathrm{m.),} \mathrm{baja}$ densidad poblacional ( 27 habitantes $/ \mathrm{km}^{2}$ de media), y un clima mediterráneo bajo influencia continental, con sequía y veranos calurosos e inviernos fríos. El promedio de lluvia oscila entre $300 \mathrm{~mm}$ y $500 \mathrm{~mm}$ y alcanza su punto máximo en primavera y en otoño.

Los datos utilizados provienen de una muestra de granjas de vacuno de leche ubicadas en la Comunidad Autónoma de Castilla y León durante los años 2016, 2017 y 2018. A la hora de seleccionar la muestra se ha procedido a estratificar las explotaciones mediante el criterio de litros de producción, de la siguiente manera: ESTRATO 1: Producción de 0 a 500.000 de litros anuales. ESTRATO 2: Producción de 500.001 a 1.000 .000 de litros anuales. ESTRA-
TO 3: Producción de más de 1.000 .000 de litros anuales.

Un $22 \%$ de las granjas corresponden al primer estrato (18 registros), un $29 \%$ al segundo (24 registros) y un $49 \%$ al tercero (40 registros). En este reparto siempre se ha tratado de ajustar la disponibilidad de datos con una distribución por estratos que sea representativa a nivel regional de acuerdo con la información proporcionada por la Junta de Castilla y León. Aunque en principio son 82 granjas, finalmente se trabaja con 80 porque hay dos con datos muy atípicos, una en Burgos y otra en León.

La información utilizada para el análisis se obtuvo mediante entrevistas personales realizadas año a año en cada una de las granjas. Todos los datos han sido facilitados por los ganaderos participantes mediante la revisión de los documentos comerciales correspondientes, de acuerdo con el reparto provincial y anual que aparece recogido en la tabla 1. Se trata de un proyecto de recogida de datos donde la participación es voluntaria, y donde la información proviene de las facturas y contabilidades (en euros y sin impuestos). Todas las explotaciones pueden ser consideradas como intensivas con predominio de la estabulación permanente.

La información de ingresos anuales se obtiene como suma de ingresos por ventas (leche, animales de desvieje y crías), subvenciones directamente relacionadas con la explotación de vacuno de leche, indemnizaciones de seguros (animales y siniestros de la propia explotación), y otros ingresos eventuales (venta de inmovilizados o similar). Este procedimiento es similar al planteado por López-Paredes y Alenda (2015).

Por el lado de los costes se utiliza el concepto de coste corrientes (CC) imputables a la explotación de vacuno de leche, y de acuerdo a lo especificado en la siguiente expresión:

$$
C C=C A+C S+M+S A+C A D
$$


Tabla 1. Reparto de granjas por provincia y año de recogida de datos. Table 1. Distribution of farms by province and year of data collection.

\begin{tabular}{lcccc}
\hline & 2016 & 2017 & 2018 & Total \\
\hline Ávila & 3 & 2 & 2 & 7 \\
Burgos & 3 & 3 & 2 & 8 \\
León & 10 & 9 & 11 & 30 \\
Palencia & 4 & 3 & 2 & 9 \\
Salamanca & 2 & 2 & 3 & 7 \\
Segovia & 1 & 2 & 1 & 4 \\
Valladolid & 2 & 2 & 5 & 9 \\
Zamora & 3 & 3 & 2 & 8 \\
\hline Total & 28 & 26 & 28 & 82 \\
\hline
\end{tabular}

Nota: no se incluye Soria por su escaso censo de vacuno lechero.

Dentro de la partida correspondiente a salarios $(S A)$ se incluyen los sueldos de todos los trabajadores remunerados. También se tiene en cuenta el coste dedicado a la alimentación del ganado tanto de producción como de recría $(C A)$, la sanidad y la reproducción (veterinario, zoosanitarios y material genético) (CS), productos para la limpieza de las instalaciones y el ganado, agua, energía y combustibles, reparaciones y conservación tanto de la maquinaria como de las instalaciones (Esto se incluye como coste de mantenimiento, M) y también los costes administrativos $(C A D)$. En el coste de alimentación se consideran las compras de alimentos de fuera de la explotación, así como el coste de producción propia, en el que se han tenido en cuenta el coste de arrendamiento de la tierra. El resto de información se recoge directamente de los datos proporcionados por los ganaderos (López-Paredes y Alenda, 2015).
Por su parte, las amortizaciones de inmovilizado (construcciones y maquinaria), se han estimado por el método de amortización lineal y valor residual cero, considerando una vida económica de 20 años para las construcciones y 10 años para la maquinaria. El coste financiero asociado al capital propio, se ha considerado también como coste de oportunidad, con un tipo de interés legal del $3 \%$ y un IPC del 1,6\% (INE, 2016). Aparte de los intereses correspondientes al capital propio (ganado, instalaciones, maquinaria y tierras propiedad de la explotación) en el coste de oportunidad también se incluye la remuneración del trabajo familiar (Acero et al., 2004)1․ El cálculo concreto del coste de oportunidad (CO) se obtiene de la siguiente expresión:

$$
\begin{gathered}
C O=K \cdot(i-I P C)+H S \cdot 115+H R \cdot 382+ \\
U C \cdot(12 \cdot 1.980)+\text { UNC } \cdot(12 \cdot 1.000)
\end{gathered}
$$

Donde $K$ es el capital invertido, $H S$ y $H R$ indican las hectáreas de secano y regadío respec-

(1) El coste de oportunidad incluye la retribución de la mano de obra familiar y se corresponde con la aportación de la mano de obra que a pesar de prestar sus servicios en la explotación no recibe remuneración por ello. 
tivamente y $115 €$ y $382 €$ son los importes medios del alquiler por hectárea de secano y regadío en Castilla y León durante los periodos objeto de este estudio según los datos consultados al Servicio de Estudios, Estadística y Planificación Agraria de la Junta de Castilla y León. Por su parte, UC y UNC se refieren a las Unidades de Trabajo Agrario (en adelante UTAs) cualificadas y no cualificadas que no reciben salario, por lo que se ha estimado un salario de los propietarios y sus familiares considerados como un coste de oportunidad mínimo cuantificado en $12.000 €$ /año por UTA en el caso de personal sin estudios y otro de

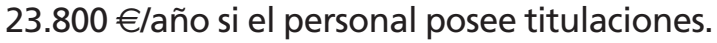
En cuanto al valor de inventario de ganado, se ha utilizado un precio de $1.272 € /$ vaca de producción, $1.390 € /$ novilla de más de 12 meses y $484 € /$ novilla de entre 3 meses y 12 meses (Observatorio de precios agrícolas y ganaderos de Castilla y León, 2020).

\section{Análisis multivariante}

La segunda parte de este epígrafe se dedica a detallar el análisis econométrico realizado. El primer paso en esta fase es identificar que variables se consideran indicadores de la rentabilidad de las explotaciones ganaderas. En este trabajo se utilizan dos medidas relativas que permiten comparar explotaciones de diferente tamaño y que se definen de la siguiente manera:

$$
\begin{aligned}
& B V_{i t}=\frac{B E_{i t}}{N V_{i t}} \\
& U V_{i t}=\frac{C C_{i t}}{P L_{i t}}
\end{aligned}
$$

Donde $B V_{i t}$ es el beneficio obtenido por la explotación $i$ en el año $t$ por cada vaca que integra la explotación animal (con independencia de que esta produzca leche o no). $N V_{i t}$ es el número de vacas y $B E_{i t}$ es el beneficio de explotación y se obtiene restando a los ingresos los costes corrientes, las amorti- zaciones del inmovilizado material y los costes de oportunidad no incluidos previamente. El beneficio por vaca es un indicador que está directamente relacionado con la rentabilidad de la explotación. Por su parte $U V_{i t}$ es el umbral de producción por vaca y recoge el número de litros que debe producir cada vaca lactante para cubrir los costes corrientes de una explotación. $C_{i t}$ recoge los costes corrientes de la explotación i en el año t y $\mathrm{PL}_{\text {it }}$ el precio de la leche obtenido por cada ganadero en ese mismo periodo de tiempo. En este caso, el umbral es un indicador de eficiencia en cuanto que, cuanto menor sea su valor, más factible será obtener rentabilidad en la granja. Hay que dejar claro que al utilizarse los costes corrientes, solo se tiene en cuenta el trabajo contratado para su cálculo y no así las UTAs no asalariadas relacionadas con propietarios y familiares. Dicho de otra manera, este umbral mide el nivel de producción de leche a partir del cual se puede comenzar a rentabilizar la mano de obra familiar y cubrir el resto de costes de oportunidad sin tener en cuenta otras fuentes de ingresos.

Se ha decidido utilizar estos dos indicadores para evaluar la rentabilidad porque ya son empleados en RENGRATI y también se tienen en cuenta por la Junta de Castilla y León en las redes de costes agrarios creadas para mejorar la competitividad de las explotaciones. Los dos se tratan de explicar a partir de un modelo econométrico del siguiente tipo:

$$
Y_{i t}=\beta X_{i t}+\varepsilon_{i t}
$$

Donde $Y_{i t}$ se refiere a cada una de las variables dependientes analizadas, $B V_{i t} \circ U V_{i t}$ respectivamente. $\beta$ es un vector de parámetros a estimar, $X_{i t}$ es un conjunto de variables explicativas y $\varepsilon_{i t}$ es un error aleatorio de media cero y varianza constante. Dentro de $X_{i t}$ se incluyen diferentes controles que son los que se piensa que pueden estar explicando la rentabilidad de cada explotación. 
El primer grupo de variables que forman parte de esos controles hace referencia a la composición de los costes de la granja, y en él se incluye el porcentaje de coste dedicado a alimentación, a sanidad o a remuneración salarial. Como alternativa a los salarios también se hacen pruebas con el coste de oportunidad que mide la aportación de los propietarios a la empresa. El segundo grupo de variables se utiliza para medir la importancia de cada uno de los componentes de los ingresos. Aquí se añaden controles que miden el porcentaje de ingresos que se debe a la venta de leche, a la venta de ganado o a subvenciones. En tercer lugar, se incluye una variable que mide el grado de tecnificación de la granja calculado como el peso que representan los costes de consumo energético sobre el total de costes corrientes y un indicador del tamaño de la granja a partir del número de vacas en producción. También se controla por algunas características de los propietarios de la granja como es el porcentaje de varones y si alguno de los propietarios tiene estudios universitarios. Finalmente se añaden controles espaciales y temporales mediante variables dicotómicas que indican la provincia a la que pertenece la granja y el año al que corresponden los datos. Estas variables toman el valor 1 en la provincia (o año) al que pertenece el dato de la granja y 0 en el resto. No obstante, hay que tener en cuenta que a la hora de realizar la estimación hay que dejar fuera una de cada grupo de variables dicotómicas para evitar problemas de multicolinealidad perfecta. Esas variables que quedan fuera son las que actúan como referencia a la hora de interpretar los coeficientes (Ávila en el caso de provincias y 2016 en el caso de los años). Las variables provinciales tienen como finalidad controlar aquellos factores diferenciales de cada territorio que no cambian con el tiempo (por ejemplo, peculiaridades de las explotaciones o diferencias climáticas). Las anuales, sin embargo, se incluyen para tener en cuenta aspectos que afectan de forma conjunta a todas las granjas y que dependen del año considerado (por ejemplo, la legislación regional, nacional o europea).

Desde un punto de vista técnico se plantea la realización de una estimación de mínimos cuadrados ordinarios donde la selección de variables se realiza a partir de su aportación al modelo por medio del estadístico t. El objetivo final es realizar un análisis "céteris páribus" que determine cuál es el efecto de una variable cuando las demás se mantienen constantes en sus valores medios. También se platea una estimación de panel que permita controlar la heterogeneidad inobservable como muestra la robustez. Dado que se trata de una muestra que pretende representar a una población superior el método adecuado para la estimación es el de mínimos cuadrados generalizados con efectos aleatorios. En ese caso nos aprovechamos de que hay un cierto seguimiento de las granjas durante tres años. No obstante, si tenemos en cuenta que todos los años algunas desaparecen y otras son nuevas, el panel es no balanceado. Para cualquier explicación adicional sobre estos métodos de estimación puede consultarse Greene (2003). Finalmente, sería conveniente comentar que todos los procedimientos estadísticos se han realizado utilizando la versión 15 del software estadístico STATA.

\section{Resultados y discusión}

De acuerdo con los datos empleados, la explotación media objeto de este estudio produce 1.423.869 L de leche/año, con un censo medio de 132 vacas, de las que un $87 \%$ están en producción, y dispone de una base territorial de 96 hectáreas donde producir alimentos para sus animales. El número de UTAs de las que dispone esta explotación es 3,65.

En relación con la procedencia de los ingresos (ver tabla 2), se puede comprobar que una media del $80 \%$ proviene de la venta de 
Tabla 2. Descriptivos relativos a las variables empleadas en el análisis.

Table 2. Descriptive relative to the variables used in the analysis.

\begin{tabular}{|c|c|c|c|c|c|}
\hline Variable & Granjas & Media & Std. Dev. & Min & Max \\
\hline Beneficio (€/vaca·año) & 80 & 487,60 & 670,69 & $-920,64$ & $2.534,58$ \\
\hline Producción Umbral (I/vaca) & 80 & $9.740,97$ & $2.006,90$ & $4.339,52$ & $15.248,19$ \\
\hline \multicolumn{6}{|c|}{ Reparto porcentual de costes por partidas } \\
\hline Alimentación & 80 & $58 \%$ & 0,10 & $35 \%$ & $79 \%$ \\
\hline Zoosanitarios & 80 & $9 \%$ & 0,04 & $2 \%$ & $24 \%$ \\
\hline Salarios & 80 & $10 \%$ & 0,08 & $0 \%$ & $30 \%$ \\
\hline C. Oportunidad & 80 & $10 \%$ & 0,13 & $0 \%$ & $60 \%$ \\
\hline Energía & 80 & $6 \%$ & 0,03 & $2 \%$ & $13 \%$ \\
\hline \multicolumn{6}{|c|}{ Reparto porcentual de ingresos por partidas } \\
\hline Leche & 80 & $80 \%$ & 0,09 & $47 \%$ & $96 \%$ \\
\hline Ganado & 80 & $7 \%$ & 0,07 & $0 \%$ & $43 \%$ \\
\hline Subvenciones & 80 & $10 \%$ & 0,04 & $1 \%$ & $20 \%$ \\
\hline \multicolumn{6}{|l|}{ Tamaño de explotación } \\
\hline $\operatorname{Vacas}\left(\mathrm{n}^{\circ}\right)$ & 80 & 132,78 & 82,39 & 24,91 & 356,67 \\
\hline \multicolumn{6}{|l|}{ Variables socioeconómicas } \\
\hline Género (\% varones) & 80 & $84 \%$ & 0,17 & $50 \%$ & $100 \%$ \\
\hline Estudios (\% universitarios) & 80 & $43 \%$ & 0,50 & $0 \%$ & $100 \%$ \\
\hline
\end{tabular}

Nota: Se han eliminado una granja de León y otra de Burgos por sus datos atípicos.

leche a industrias (96\% en el caso más extremo), siendo un resultado lógico si tenemos en cuenta que se trata de granjas lecheras. El resto de los ingresos se reparte entre un $7 \%$ por venta de ganado y un $10 \%$ de subvenciones. Estos datos descriptivos son similares a los encontrados por Álvarez et al. (2016) en un estudio realizado sobre una muestra de 5 granjas.

Respecto a los costes corrientes, se observa que el $58 \%$ se corresponde con la alimentación de los animales, obtenida como la suma entre alimentos comprados y alimentos producidos, y puede alcanzar casi el $80 \%$ en al- gún caso. Por su parte, tanto los costes sanitarios y de reproducción del ganado, como los salarios o el coste de oportunidad suponen un gasto medio cercano al $10 \%$ (Un resumen de los costes e ingresos medios por vaca desagregado por partidas y estratos se puede consultar en la tabla 3).

De acuerdo con los datos recogidos en la tabla 2 , también se puede destacar que el beneficio medio por granja de la muestra se sitúa en valores cercanos a los $500 €$ /vaca y es necesario que cada vaca produzca un promedio de casi $10.000 \mathrm{~L}$ de leche/año para cubrir costes. Pero más allá de estos resultados medios se obser- 
Tabla 3. Valores medios de las diferentes partidas de ingresos y costes por estrato de producción y año en $€ /$ vaca.

Table 3. Average values of the different items of income and costs by production stratum and year in €lcow.

\begin{tabular}{|c|c|c|c|c|c|c|c|c|c|}
\hline & \multicolumn{3}{|c|}{2016} & \multicolumn{3}{|c|}{2017} & \multicolumn{3}{|c|}{2018} \\
\hline & $\begin{array}{c}\text { Estrato } \\
1\end{array}$ & $\begin{array}{c}\text { Estrato } \\
2\end{array}$ & $\begin{array}{c}\text { Estrato } \\
3\end{array}$ & $\begin{array}{c}\text { Estrato } \\
1\end{array}$ & $\begin{array}{c}\text { Estrato } \\
2\end{array}$ & $\begin{array}{c}\text { Estrato } \\
3\end{array}$ & $\begin{array}{c}\text { Estrato } \\
1\end{array}$ & $\begin{array}{c}\text { Estrato } \\
2\end{array}$ & $\begin{array}{c}\text { Estratc } \\
3\end{array}$ \\
\hline \multicolumn{10}{|l|}{ INGRESOS } \\
\hline Leche & 2.308 & 2.835 & 3.390 & 2.485 & 2.950 & 3.476 & 2.958 & 3.094 & 3.835 \\
\hline Ganado & 328 & 136 & 178 & 377 & 271 & 195 & 356 & 342 & 305 \\
\hline Subvenciones & 426 & 401 & 319 & 465 & 472 & 359 & 490 & 392 & 367 \\
\hline \multicolumn{10}{|l|}{ COSTES } \\
\hline Alimentación & 1.480 & 1.773 & 1.821 & 1.431 & 1.514 & 1.527 & 1.916 & 1.911 & 1.948 \\
\hline Zoosanitarios & 204 & 208 & 329 & 179 & 180 & 253 & 197 & 278 & 381 \\
\hline Energía & 154 & 190 & 165 & 131 & 170 & 139 & 160 & 203 & 209 \\
\hline Salarios & 198 & 212 & 352 & 400 & 311 & 433 & 194 & 336 & 460 \\
\hline Amortizaciones & 307 & 349 & 268 & 230 & 300 & 251 & 275 & 316 & 266 \\
\hline Coste de oportunidad & 515 & 206 & 138 & 326 & 200 & 90 & 572 & 269 & 116 \\
\hline
\end{tabular}

van grandes oscilaciones, hay granjas con pérdidas cercanas a los $1.000 € /$ vaca y otras que superan los $2.500 € /$ vaca de beneficio. También se encuentran explotaciones que con producir algo más de $4.000 \mathrm{l} / \mathrm{vaca}$ cubren costes mientras otras necesitarían superar los $15.000 \mathrm{l} / \mathrm{vaca}$, esta variabilidad concuerda con la reseñada por Pérez-Carbajal y Alenda et al. (2003).

Otra variable importante es el gasto en energía que muestra en cierta medida el grado de tecnificación de la explotación y cuyo peso, en el total de costes, oscila entre el $2 \%$ y el $13 \%$ con una media del $6 \%$. En cuanto al tamaño, las granjas tienen 132 vacas como media, pero hay explotaciones que superan las 350 vacas y otras que no llegan a las 25 vacas. Las dos últimas variables se refieren a características personales de los trabajadores en las granjas, y muestran que en su mayoría los empleados son varones y que un $43 \%$ de los propietarios tiene estudios universitarios.

En la segunda parte de los resultados se muestran los coeficientes asociados a las estimaciones planteadas en el análisis multivariante. Como ya se ha comentado, se utilizan dos estimaciones, una sobre el beneficio medido en euros por vaca, y otra sobre el umbral de producción calculado como el número de litros que debe producir cada vaca para cubrir los costes corrientes de la explotación. En ambos casos se estiman cuatro modelos distintos que se van modificando con el fin de obtener la mejor especificación posible, de acuerdo al valor que proporciona el ajustado.

El primero de los modelos utiliza como variables explicativas, además de los controles temporales y espaciales, los componentes de costes como el peso de la alimentación, el de 
los zoosanitarios y el de la masa salarial. El segundo completa al anterior con variables de ingresos como el peso de la venta de leche, el peso de la venta de ganado o la importancia de las subvenciones. En el modelo III se añaden variables como el indicador de mecanización, el número de vacas de la granja y variables socioeconómicas como el porcentaje de varones y el nivel de estudios del propietario. Finalmente, el último modelo sustituye el peso de la masa salarial por el coste de oportunidad y elimina las variables socioeconómicas. En el caso del umbral por vaca se modifica un poco este último modelo ya que mantiene el nivel de estudios porque resultaba significativo y elimina la variable mecanización y el control de tamaño porque no eran explicativas en el modelo III. En esa selección de variables también se tiene en cuenta su propia significación individual a través del estadístico t de Student.

Los resultados de la tabla 4 muestran un efecto positivo y significativo tanto del gasto en alimentación, similar a lo descrito por Priyamvada y Mishra (2019), como en zoosanitarios, sobre el beneficio obtenido por vaca. Dicho de otra manera, el invertir en vacas más sanas y mejor alimentadas genera más rentabilidad a la explotación. También se aprecia un mayor beneficio cuando adquieren más importancia los salarios y una reducción cuando se incrementa el peso de los costes de oportunidad. Este resultado parece indicar una mayor rentabilidad de las explotaciones donde el trabajo no se concentra en los propietarios, y seguramente este asociado a una mayor profesionalización de la empresa. Por el lado de los ingresos, la única variable relevante son los ingresos por venta de ganado que afectan positivamente al nivel de beneficios. Este resultado parece indicar que una mayor diversificación de los ingresos también parece mejorar la situación de la explotación.

Los modelos III y IV reflejan un efecto positivo de la tecnificación y del tamaño de la explotación sobre el nivel de rentabilidad. Este resultado vuelve a ser coherente con el resultado obtenido por los salarios y que se relacionaba con el hecho de que una mayor profesionalización redunda en una mayor rentabilidad. Nehring et al. (2016) encuentran resultados similares en un estudio realizado para granjas de Estados Unidos y la Unión Europea y Sinisalo (2015) para otro realizado con explotaciones de Finlandia. Las variables referidas a la composición por género y al nivel educativo de los propietarios de la explotación del modelo III no resultan relevantes para la estimación del modelo. Finalmente, los controles temporales reflejan un incremento de los beneficios por vaca en 2017, y los controles espaciales muestran que tienen más beneficio las granjas situadas en Burgos, Salamanca o Zamora².

En términos de significación global se puede comprobar como todos los modelos explican más del $40 \%$ de la variabilidad de la variable dependiente. En algunos casos, como en la especificación III, esa significación supera el $50 \%$.

Los resultados correspondientes al umbral de producción por vaca se presentan en la tabla 5 . En este caso se comprueba que dicho umbral crece cuando lo hace el porcentaje de gasto dedicado a alimentación, zoosanitarios o salarios. Como en el caso anterior también se han hecho pruebas sustituyendo la variable que mide el peso de los salarios por la correspondiente al coste de oportunidad. Ahora el coste de oportunidad vuelve a tener un signo negativo y significativo. Este resul-

(2) Los coeficientes de estas variables no se incluyen en la tabla pero están a disposición de los interesados previa petición a los autores. 
Tabla 4. Estimación de los beneficios por vaca.

Table 4. Estimate of benefits per cow.

\begin{tabular}{|c|c|c|c|c|c|c|c|c|}
\hline & \multicolumn{8}{|c|}{ Especificaciones } \\
\hline & \multicolumn{2}{|c|}{ I } & \multicolumn{2}{|c|}{ II } & \multicolumn{2}{|c|}{ III } & \multicolumn{2}{|c|}{ IV } \\
\hline & Coef. & $t$ & Coef. & $t$ & Coef. & $t$ & Coef. & $t$ \\
\hline \multicolumn{9}{|l|}{ Variables de coste } \\
\hline Alimentación & $2.107,6$ & 2,35 & $1.829,3$ & 1,96 & $3.374,0$ & 3,4 & $1.090,8$ & 1,37 \\
\hline Zoosanitarios & $7.617,8$ & 3,66 & $7.155,4$ & 3,43 & $7.723,3$ & 3,94 & $5.820,3$ & 2,92 \\
\hline Coste Oportunidad & & & & & & & $-1.286,4$ & $-1,93$ \\
\hline Salarios & $4.325,3$ & 3,98 & $3.836,4$ & 3,41 & $4.543,8$ & 3,63 & & \\
\hline Mecanización & & & & & $6.410,8$ & 2,34 & $2.762,8$ & 1,07 \\
\hline \multicolumn{9}{|l|}{ Variables de ingresos } \\
\hline Venta leche & & & $-74,2$ & $-0,05$ & 93,5 & 0,06 & 44,2 & 0,03 \\
\hline Venta ganado & & & $1.412,0$ & 0,94 & $2.582,7$ & 1,68 & $2.677,8$ & 1,74 \\
\hline Subvención & & & $-2.885,9$ & $-1,23$ & 214,8 & 0,08 & $-455,2$ & $-0,18$ \\
\hline $\mathrm{N}^{\circ}$ Vacas & & & & & 2,8 & 2,93 & 2,6 & 2,64 \\
\hline \multicolumn{9}{|c|}{ Variables socioeconómicas } \\
\hline Varones & & & & & $-2.71,2$ & $-0,64$ & & \\
\hline Estudios & & & & & $-2.16,3$ & $-1,59$ & & \\
\hline \multicolumn{9}{|c|}{ Controles temporales (el año 2016 es la referencia) } \\
\hline Año 2017 & 542,4 & 3,60 & 543,6 & 3,55 & 517,6 & 3,62 & 577,7 & 3,82 \\
\hline Año 2018 & $-26,9$ & $-0,19$ & $-49,4$ & $-0,35$ & $-52,1$ & $-0,39$ & $-57,7$ & $-0,4$ \\
\hline Control provincial & \multicolumn{2}{|c|}{$\mathrm{Si}$} & \multicolumn{2}{|c|}{$\mathrm{Si}$} & \multicolumn{2}{|c|}{$\mathrm{Si}$} & \multicolumn{2}{|l|}{$\mathrm{Si}$} \\
\hline Constante & $-2.687,6$ & -3.35 & -2097.3 & -1.30 & $-3.985,7$ & -2.43 & $-1.685,12$ & $-1,08$ \\
\hline$R^{2}$ & 0,51 & 0,55 & 0,63 & 0,57 & & & & \\
\hline$R^{2}$ ajustado & 0,43 & 0,44 & 0,52 & 0,45 & & & & \\
\hline Datos & \multicolumn{8}{|c|}{$80 *$} \\
\hline
\end{tabular}

Notas: En negrita aparecen los coeficientes significativos a un mínimo del $10 \%$.

* Se han eliminado una granja de León y otra de Burgos por sus datos atípicos.

tado indica que cuando es mayor el peso del trabajo correspondiente a los propietarios de la explotación son necesarios menos litros por vaca para cubrir los costes de la empresa.
O también, que una mayor profesionalización de la empresa incrementa el número de litros que deben obtenerse por vaca para poder compensar el aumento de costes. 
Tabla 5. Estimación de la producción umbral por vaca de lactación.

Table 5. Estimation of the threshold production per lactating cow.

\begin{tabular}{|c|c|c|c|c|c|c|c|c|}
\hline & \multicolumn{8}{|c|}{ Especificaciones } \\
\hline & \multicolumn{2}{|c|}{ I } & \multicolumn{2}{|c|}{ II } & \multicolumn{2}{|c|}{ III } & \multicolumn{2}{|c|}{ IV } \\
\hline & Coef. & $t$ & Coef. & $t$ & Coef. & $t$ & Coef. & $t$ \\
\hline \multicolumn{9}{|l|}{ Variables de coste } \\
\hline Alimentación & $6.161,5$ & 1,86 & $5.896,5$ & 1,75 & $5.609,5$ & 1,45 & $1.850,2$ & 0,70 \\
\hline Zoosanitarios & $17.584,8$ & 2,29 & $15.391,7$ & 2,04 & $13.428,4$ & 1,76 & $10.916,4$ & 1,53 \\
\hline Coste Oportunidad & & & & & & & $-4.861,6$ & 2,25 \\
\hline Salarios & $11.095,1$ & 2,77 & $9.642,1$ & 2,37 & $7.853,3$ & 1,61 & & \\
\hline Mecanización & & & & & $-1.897,5$ & $-0,18$ & & \\
\hline \multicolumn{9}{|l|}{ Variables de ingresos } \\
\hline Venta leche & & & $-11.843,0$ & $-2,22$ & $-13.790,0$ & $-2,46$ & $-12.403,2$ & $-2,31$ \\
\hline Venta ganado & & & $-7.888,0$ & $-1,45$ & $-9.381,1$ & $-1,57$ & $-7.855,7$ & $-1,38$ \\
\hline Subvención & & & $-22.683,4$ & $-2,67$ & $-22.482,3$ & $-2,14$ & $-21.854,7$ & $-2,40$ \\
\hline $\mathrm{N}^{\circ}$ Vacas & & & & & 2,8 & 0,77 & & \\
\hline \multicolumn{9}{|c|}{ Variables socioeconómicas } \\
\hline Varones & & & & & $-566,0$ & $-0,34$ & & \\
\hline Estudios & & & & & 918,1 & 1,73 & $1.170,8$ & 3,31 \\
\hline \multicolumn{9}{|c|}{ Controles temporales (el año 2016 es la referencia) } \\
\hline Año 2017 & $-91,3$ & $-0,16$ & 209,6 & 0,38 & 300,7 & 0,54 & 370,2 & $0,68 *$ \\
\hline Año 2018 & 839,8 & 1,62 & $1.053,8$ & 2,04 & $1.058,9$ & 2,01 & $1.110,9$ & 2,20 \\
\hline Control provincial & \multicolumn{2}{|c|}{ Si } & \multicolumn{2}{|c|}{ Si } & \multicolumn{2}{|c|}{ Si } & \multicolumn{2}{|c|}{$\mathrm{Si}$} \\
\hline Constante & $3.724,5$ & 1,26 & $16.729,2$ & 2,87 & $19.183,3$ & 3,01 & $21.416,5$ & 3,81 \\
\hline$R^{2}$ & 0,26 & 0,34 & 0,38 & 0,37 & & & & \\
\hline$R^{2}$ ajustado & 0,13 & 0,18 & 0,19 & 0,22 & & & & \\
\hline Datos & \multicolumn{8}{|c|}{$80 *$} \\
\hline
\end{tabular}

Notas: En negrita aparecen los coeficientes significativos a un mínimo del $10 \%$.

* Se han eliminado una granja de León y otra de Burgos por sus datos atípicos.

De acuerdo con los modelos II, III y IV cuanto mayor peso tenga la venta de leche y las subvenciones en los ingresos menor es el margen necesario para cubrir costes. Sin embargo, ni el nivel de mecanización ni el tamaño de la empresa resultaron estadísticamente significativos. Como en el caso anterior, el género sigue sin tener efecto y el nivel de estudios del 
propietario no solo no reduce el margen, sino que lo incrementa. Este resultado puede ser consecuencia de que una mayor formación del propietario está asociada a un mayor coste de oportunidad elevando también el margen necesario para cubrir costes.

Finalmente, los controles temporales indican un mayor margen en 2018 y también en las provincias de León, Valladolid y Zamora, aunque este último resultado solo se produce con las especificaciones III y IV. En términos de significatividad, se comprueba que los modelos que explican el umbral de producción son menos significativos llegando solo a explicar el $20 \%$ de la varianza de la variable dependiente (umbral de producción).

Para terminar, y como medida de robustez, se han realizado pruebas utilizando una estimación de panel no balanceado con efectos aleatorios para los modelos III y IV y para las dos variables objetos de estudio. En este caso todos los resultados obtenidos vuelven a ser coherentes con los presentados a través de la estimación de mínimos cuadrados ordinarios (pueden consultarse en la tabla 6).

A modo de resumen, los efectos positivos asociados a los costes en alimentación y zoosanitarios en los dos indicadores empleados tienen dos interpretaciones. Por un lado, una mayor inversión en alimentación y sanidad incrementa el coste de producción y por lo tanto también aumenta el margen que se debe obtener por vaca para poder hacerles frente. Por otro, esa mejor alimentación y sanidad de los animales incrementa su producción. En ese sentido, el efecto positivo de estas variables sobre el beneficio por vaca pone de manifiesto que el incremento de leche compensa el aumento de costes generado y por lo tanto también la rentabilidad de la explotación.

Otras dos variables importantes que actúan en sentido opuesto son el peso de la masa salarial y el coste de oportunidad. Tanto en el caso del beneficio por vaca como en el umbral de producción la masa salarial tiene un signo positivo y el coste de oportunidad negativo. Esta evolución contraria puede ser consecuencia de que se trata de factores sustitutivos ya que el coste de oportunidad se relaciona con el empleo de recursos propios y trabajo no remunerado. Siguiendo esta línea de análisis, un mayor peso del trabajo remunerado y menor aportación de los propietarios, es compatible con una mayor profesionalización de la empresa. En estos casos es necesaria una mayor inversión y por lo tanto aumenta el margen de producción necesario para compensarla. Pero por otro lado, el rendimiento de esa inversión tiene un efecto multiplicador que hace que el incremento de ingresos compense los gastos y por lo tanto aumente el beneficio.

Este efecto positivo de la profesionalización de la empresa también se ve reflejado en el signo positivo y significativo tanto del gasto en energía como del tamaño de la empresa sobre el beneficio. La primera de esas variables indica que un mayor gasto energético, seguramente asociado a un mayor empleo de tecnología, suele redundar en una mayor rentabilidad de la empresa. La segunda está más relacionada con la existencia de economías de escala. Los incrementos de tamaño de las empresas, aunque incrementan los costes totales, parecen generar un incremento más que proporcional en los ingresos que mejora la rentabilidad de la explotación. En general se aprecia que toda inversión en una dirección más profesional y en el bienestar de los animales, aunque puede generar aumento de costes, también da lugar a una mejora de los ingresos que compensa la inversión y mejora los beneficios de la empresa.

Finalmente, las variables socioeconómicas como la composición por sexo de los trabajadores o el nivel de estudios del propietario no parecen afectar a los resultados de la empresa. El único efecto reseñable lo encontramos para el caso del nivel de estudios en el 
Tabla 6. Resultados de la estimación de panel no balanceado (Mínimos Cuadrados Generalizados). Table 6. Results of the estimation of the unbalanced panel (Generalized Least Squares).

\begin{tabular}{|c|c|c|c|c|c|c|c|c|}
\hline & \multicolumn{4}{|c|}{ Beneficio } & \multicolumn{4}{|c|}{ Umbral de producción } \\
\hline & \multicolumn{2}{|c|}{1} & \multicolumn{2}{|c|}{ II } & \multicolumn{2}{|c|}{ III } & \multicolumn{2}{|c|}{ IV } \\
\hline & Coef. & $t$ & Coef. & $t$ & Coef. & $t$ & Coef. & $t$ \\
\hline \multicolumn{9}{|l|}{ Variable de coste } \\
\hline Alimentación & $3.302,6$ & 3,30 & $1.076,2$ & 1,33 & $4.912,4$ & 1,28 & $1.356,6$ & 0,52 \\
\hline Zoosanitarios & $7.372,1$ & 3,66 & $5.186,4$ & 2,48 & $11.667,5$ & 1,47 & $8.821,4$ & 1,21 \\
\hline Coste Oportunidad & & & $-1.266,0$ & $-1,75$ & & & $-6.437,5$ & $-2,70$ \\
\hline Salarios & $4.575,0$ & 3,56 & & & $7.476,1$ & 1,45 & & \\
\hline Mecanización & $6.213,7$ & 2,24 & $2.901,9$ & 1,08 & $1.359,7$ & $-0,13$ & & \\
\hline \multicolumn{9}{|l|}{ Variables de ingresos } \\
\hline Venta leche & 92,22 & 0,06 & $-97,0$ & $-0,07$ & $-12.633,4$ & $-2,32$ & $-11.778,9$ & $-2,38$ \\
\hline Venta ganado & $2.766,6$ & 1,78 & $2.898,1$ & 1,82 & $-8.545,1$ & $-1,43$ & $-7.289,7$ & $-1,32$ \\
\hline Subvención & $-221,3$ & $-0,08$ & $-1.113,9$ & $-0,43$ & $-20.132,2$ & $-1,97$ & $-18.009,8$ & $-2,04$ \\
\hline $\mathrm{N}^{\circ}$ Vacas & 2,5 & 2,58 & 2,4 & 2,18 & 3,6 & 0,88 & & \\
\hline \multicolumn{9}{|c|}{ Variables socioeconómicas } \\
\hline Varones & $-207,6$ & $-0,48$ & & & $-560,6$ & $-0,34$ & & \\
\hline Estudios & $-207,7$ & $-1,44$ & & & 842,3 & 1,38 & $1.173,6$ & 1,88 \\
\hline \multicolumn{9}{|c|}{ Controles temporales (el año 2016 es la referencia) } \\
\hline Año 2017 & 513,2 & 3,69 & 568,0 & 3,99 & 218,4 & 0,43 & 206,4 & 0,45 \\
\hline Año 2018 & $-54,9$ & $-0,41$ & $-57,3$ & $-0,42$ & 881,8 & 1,79 & 892,9 & 1,99 \\
\hline Control provincial & \multicolumn{2}{|c|}{ Si } & \multicolumn{2}{|c|}{ Si } & \multicolumn{2}{|l|}{ Si } & \multicolumn{2}{|l|}{$\mathrm{Si}$} \\
\hline Constante & $-3892,0$ & $-2,35$ & $-1.446,7$ & $-0,92$ & $18.476,5$ & 2,88 & $20.939,6$ & 4,01 \\
\hline Datos & \multicolumn{8}{|c|}{80 * } \\
\hline
\end{tabular}

Notas: En negrita aparecen los coeficientes significativos a un mínimo del $10 \%$.

* Se han eliminado una granja de León y otra de Burgos por sus datos atípicos.

umbral de producción. En concreto se aprecia que a mayor formación mayor será el coste de oportunidad repercutido, y por lo tanto la explotación deberá obtener un margen superior. No obstante, esto no afecta sobre los beneficios seguramente porque esa mayor formación también ayuda a una mejor dirección de la empresa. 


\section{Conclusiones}

Todos los incrementos de gasto destinados a mejorar la alimentación y los cuidados veterinarios tienen un efecto positivo sobre la rentabilidad de las explotaciones.

Aquellas empresas donde los ingresos por leche son más importantes y donde las subvenciones tienen más peso tienen un umbral de producción por vaca más bajo. Sin embargo, cuando los ingresos se diversifican con la venta de carne son mayores los beneficios por vaca.

La mayor profesionalidad de las explotaciones traducida en un mayor peso de la masa salarial y en una reducción de la aportación del propietario, incrementa tanto el umbral de producción como los beneficios por vaca, mejorando así la rentabilidad de la explotación.

\section{Agradecimientos}

A Dña. M ${ }^{a}$ Ángeles Muñoz Lebrato, Jefa del Servicio de Producción Ganadera de la Junta de Castilla y León, por su apoyo personal y aportaciones a esta publicación.

\section{Referencias bibliográficas}

Acero R, García A, Ceular N, Artacho C, Martos J (2004). Aproximación metodológica a la determinación de costes en la empresa ganadera. Archivos de zootecnia 53(201): 91-94.

Álvarez A, Cabrera VE, Heras J (2016). Rangos de rentabilidad de explotaciones pequeñas y medianas de vacuno lechero en el noroeste de España durante el año 2015. Boletín Anembe 112: $26-28$

ECREA, Estudio de Costes y Rentas de las Explotaciones Agrarias (2016). Disponible online en: https://www.mapa.gob.es/es/ministerio/servicios/analisis-y-prospectiva/ECREA-Informes_Ganaderia.aspx. (Consultado: mayo 2020).
ECREA, Estudio de Costes y Rentas de las Explotaciones Agrarias (2017). Disponible online en: https://www.mapa.gob.es/es/ministerio/servicios/analisis-y-prospectiva/ECREA-Informes_Ganaderia.aspx. (Consultado: mayo 2020).

Greene WH (2003). Econometric analysis. Pearson Education India.

INE (2016). Renta agraria. Disponible en: https:// www.ine.es/prodyser/pubweb/anuario16/anu16 _12agric.pdf (consultado: 24 noviembre 2020).

INE (2018). Renta agraria. Disponible en: https:// www.ine.es/prodyser/pubweb/anuario18/anu18 _12agric.pdf (consultado: 24 noviembre 2020).

López-Paredes J, Alenda R (2015). Impacto de la mejora de la productividad en la rentabilidad del sector vacuno de carne en España. XVI Jornadas sobre producción animal, 19 y 20 de mayo, Zaragoza, España, pp. 531-533.

MAPA (2019). Informe de coyuntura del sector vacuno de leche, Subdirección General de Productos Ganaderos, Dirección General de Producciones y Mercados Agrarios. Disponible online en: https://www.mapa.gob.es/ca/agricul tura/estadisticas/informedecoyunturasectorvacunodeleche-abril2019_tcm34-58835.pdf (Consultado: mayo 2020).

MAPA (2020). Estructura del sector vacuno lechero en España y en la unión europea 2015-2018, Subdirección General de Productos Ganaderos, Dirección General de Producciones y Mercados Agrarios. Disponible online en: https://www. mapa.gob.es/es/ganaderia/temas/producciony-mercados-ganaderos/estructuraysituaciondelsectorvacunolecheroenespanayenlaue_versionfinal_29enero_tcm30-524899.pdf (Consultado: mayo 2020).

Milán MJ, Frendi F, González-González R, Caja G (2014). Cost structure and profitability of Assaf dairy sheep farms in Spain. Journal of Dairy Science 94: 771-784. https://doi.org/10.3168/jds. 2013-7884

Nehring R, Sauer J, Gillespie J, Hallahan C (2016). United States and European Union dairy farms: where is the competitive edge? International Food and Agribusiness Management Review 19(B): 219-239. http://dx.doi.org/10.22004/ag. econ.244638 
Observatorio de precios agrícolas y ganaderos de Castilla y León (2020). Precios de productos ganaderos. Disponible online en: https://agriculturaganaderia.jcyl.es/web/es/estadistica-informacion-agraria/precios-productos-ganaderos.ht ml. (Consultado: mayo 2020).

Pérez-Cabal MA, Alenda R (2003). El carácter rentabilidad en vacuno de leche. $X$ Jornadas sobre Producción Animal, 14-19 de mayo, Zaragoza, España, pp. 582-584.

Priyamvada S, Mishra BK (2019). Economic analysis of dairy cattle farms under town milk supply scheme in Jorhat district of Assam. Indian Journal of Dairy Science 72(3): 318-327. https://doi. org/10.33785/IJDS.2019.v72i03.013
RENGRATI (2018). Panel Nacional de Vacuno de leche. Ministerio de agricultura pesca y alimentación. Disponible online en: https://www. mapa.gob.es/es/ganaderia/temas/producciony-mercados-ganaderos/sectores-ganaderos/redde-granjas-tipicas/vacuno-lechero/. (Consultado: mayo 2020).

Sinisalo A (2015). Production costs of Finnish dairy farms in the 2000s. Economic Science for Rural Development 37: 26-34.

(Aceptado para publicación el 24 de noviembre de 2020) 